\title{
Design, Fabrication and Performance of the Silicon Charge Detector for the ISS-CREAM
}

\author{
Jik Lee ${ }^{1}$ (for the ISS-CREAM Collaboration ${ }^{2}$ ) \\ Sungkyunkwan University \\ E-mail: jiklee999@gmail.com
}

The ISS-CREAM experiment is a space-borne mission designed for the precision measurement of energy and elemental composition of cosmic rays. It will be launched to the International Space Station. The Silicon Charge Detector (SCD) for the ISS-CREAM experiment is an instrument equipped with four layers of high-precision silicon pixel sensors and readout electronics arranged in such a manner that it is free of dead area. Therefore the SCD is capable of the precise measurement of elemental composition of cosmic rays with the charge resolution of $\delta Z \leq 0.2 e$ for proton $(\mathrm{Z}=1)$ to nickel $(\mathrm{Z}=28)$. We present the design and fabrication of the SCD. The SCD successfully passed the space environment test, namely thermal-vacuum and vibration tests, at the instrumentlevel. The SCD has been integrated into the ISS-CREAM payload, and the payload currently undergoes a series of space environment tests at the system-level. We present the performance of the SCD checked in-between the space environment tests at the system-level.

The 34th International Cosmic Ray Conference,

30 July- 6 August, 2015

The Hague, The Netherlands

\footnotetext{
${ }^{1}$ Speaker

${ }^{2} \mathrm{http} / / /$ cosmicray.umd.edu/iss-cream/collaboration
} 


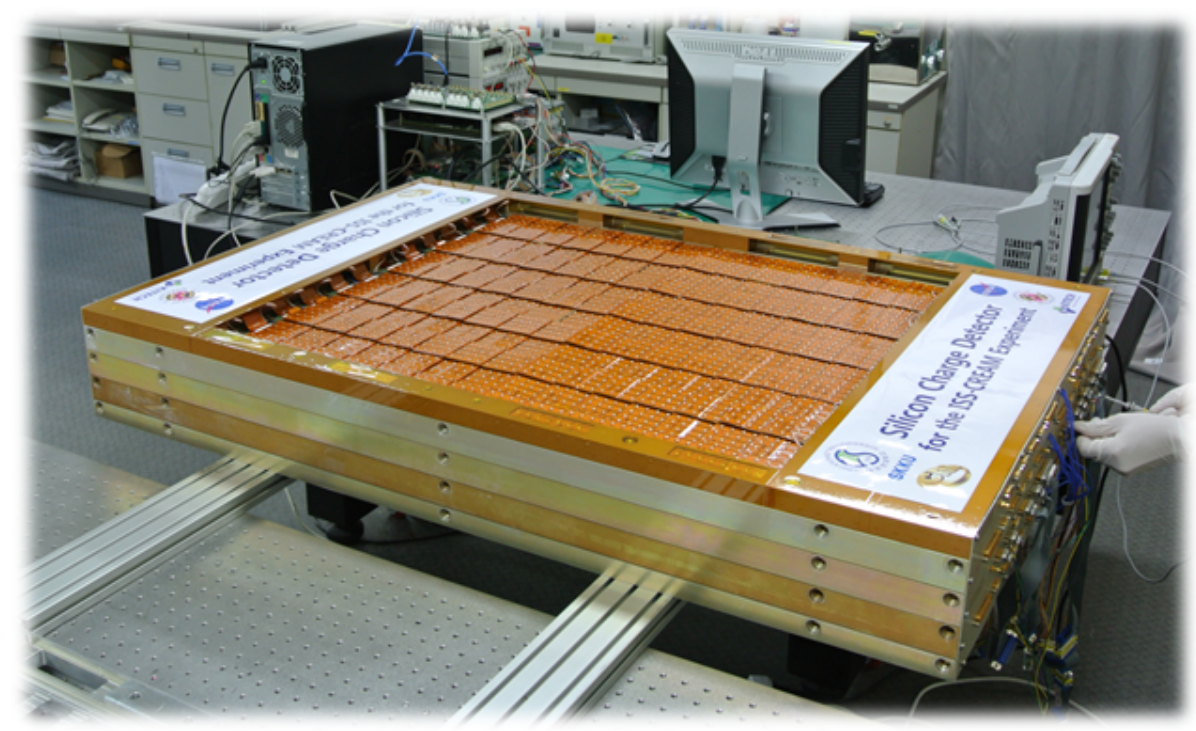

Figure 1: Overall view of SCD.

\section{Introduction}

The Antarctica balloon-borne experiment Cosmic Ray Energetics And Mass (CREAM) has successfully completed six flights between the years 2004 and 2010, and it collected total data of 161-day exposure during these flights. It observed the unexpected discrepant hardening in energy spectra of comic rays [1]. However, high-statistics data of energetic cosmic rays are required for the further investigation of the unexpected hardening in comic-ray energy spectra. The International Space Station (ISS) is an ideal platform to further investigate the unexpected hardening as well as explore the fundamental issues like the acceleration mechanism and the origin of energetic cosmic rays because of the high duty cycle of the experiment in the ISS platform. Then the space-borne experiment ISS-CREAM stationed at the ISS was an obvious next step towards further investigation and exploration of the outstanding issues with high-statistics. The ISS-CREAM experiment adapts the detector concept and technology proven at the balloon-borne CREAM experiment.

The ISS-CREAM payload is configured with a finely segmented four-layer Silicon Charge Detector for charge measurements and a sampling tungsten/scintillator calorimeter including a carbon target for energy measurements. These detectors have already demonstrated their capabilities to determine the charge and energy of high-energy cosmic rays from $10^{10}$ to $>10^{14} \mathrm{eV}$ for the proton to iron elemental range with excellent resolution. In addition, two new compact detectors are installed: Top/Bottom Counting Detectors (TCD/BCD) and Boronated Scintillator Detector (BSD). The TCD and BCD each consist of a plastic scintillator and 400 photodiodes. The TCD is located between the carbon target and the calorimeter. The BSD consists of a boron-loaded scintillator read out by photomultiplier tubes which detects late neutrons produced by particle interactions in the calorimeter. The BSD is located at the bottom of the instrument stack beneath the BCD. The configuration of the ISS-CREAM payload and details of its components are discussed elsewhere [2]. 


\section{Design and Fabrication of the Silicon Charge Detector}

The SCD for the ISS-CREAM experiment (ISS-CREAM SCD) consists of silicon pixel sensors, analog electronics, digital electronics and supporting mechanical structure. An overall view of the completed four-layer SCD is shown in Fig. 1. The weight of the SCD is $143 \mathrm{~kg}$, and its outermost dimensions are $1227 \mathrm{~mm}$ (length) x $817 \mathrm{~mm}$ (width) x $166 \mathrm{~mm}$ (height). The SCD has four layers, and the active area in each layer is $818 \mathrm{~mm}$ x $818 \mathrm{~mm}$. Each layer has 2688 channels and therefore the SCD has total 10752 channels. The power consumption of the SCD is $182.5 \mathrm{~W}$. Details of the SCD building blocks mentioned above are described in the following subsections. The single layer layout of the SCD is shown in Fig. 2. How the single layer of the SCD is built from these building blocks of the SCD and how the four-layer SCD is assembled are also explained in the following sections.

All components of the ISS-CREAM SCD and the balloon-borne CREAM SCD have the exact same functionality. However, the components of the ISS-CREAM SCD are all space-qualified. Especially all electronics components of the ISS-CREAM SCD are radiation-hard while a lot of electronics components of the balloon-borne CREAM SCD are not radiation-hard. The singlelayer mechanical structure of the ISS-CREAM SCD has less material in the active area than the structure of the balloon-borne CREAM SCD.

\subsection{Silicon Sensor}

Silicon pixel sensors in the ISS-CREAM SCD are fabricated on 525- $\mu \mathrm{m}$ thick silicon wafers. Each silicon sensor has 16 pixels, and it is attached to a flexible printed circuit board for signal readout inter-connection. The process for fabricating such silicon sensors and completing the signal readout inter-connection is explained elsewhere [3]. Silicon sensors in general are known to be able to tolerate radiation level in space so that they exhibit no sign of degradation in their functionality even after many years of exposure to the radiation in space.

\subsection{Analog Electronics}

Analog electronics boards are equipped with CR1.4 ASIC chips which convert charge signals from silicon sensors to voltage signals [4]. The CR1.4 chips have been used in the calorimeter of the satellite-borne PAMELA experiment for nine years [5]. Therefore the radiation hardness of the CR1.4 chips has been proved by the space heritage of the PAMELA experiment. The front side of an analog board has connectors and mounting holes to accommodate seven silicon sensors while the backside of the board has seven CR1.4 chips.

\subsection{Ladder}

Seven silicon sensors will be mounted onto the front side of an analog board to form a ladder. Note that the silicon sensors in the ladder will be slightly overlapped with each other so that there is no dead area along the length of the ladder. A ladder is a basic mechanical unit that populates the single layer structure of the SCD. Also a ladder is a basic electrical unit to be powered on/off and monitored. Various voltage, current and temperature sensors are installed in each ladder. The status and condition of the ladder is monitored by the slow control system (called housekeeping 


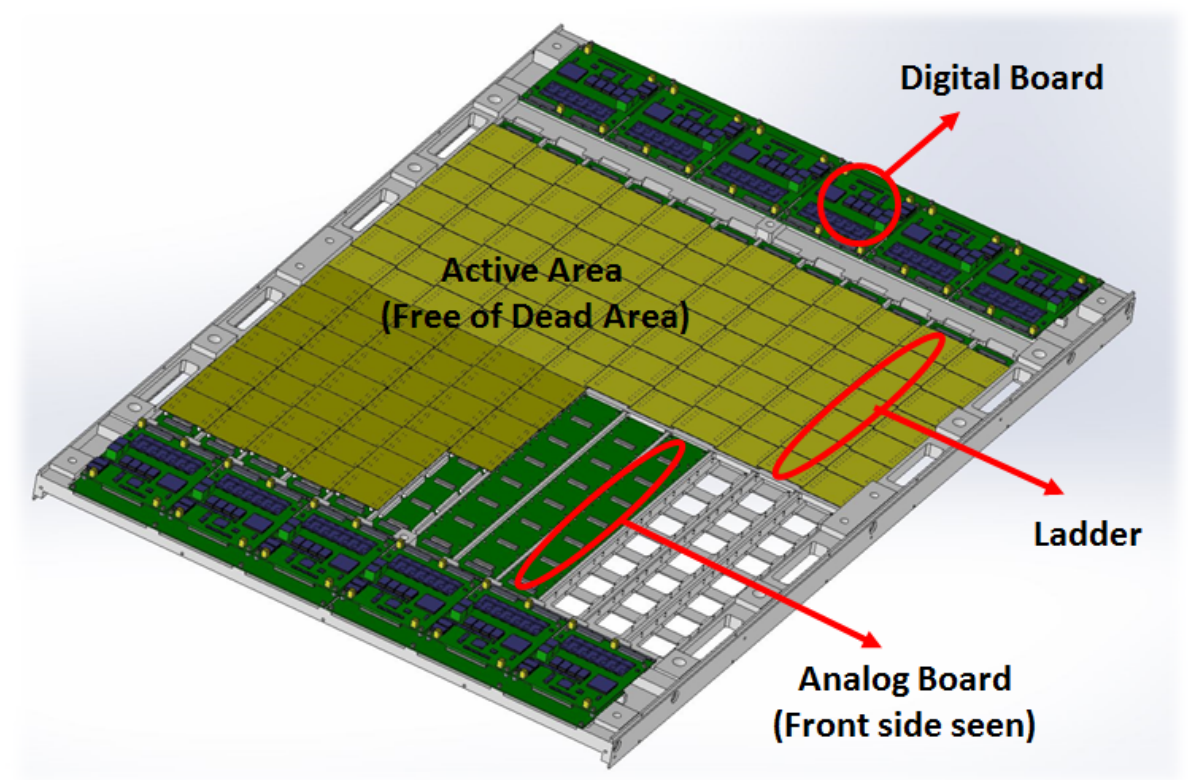

Figure 2: SCD single layer layout.

system) of the ISS-CREAM experiment which carries out constant read-out and digitization of analog signals from those sensors.

\subsection{Digital Electronics}

Digital electronics boards are equipped with various voltage regulators, radiation-hard Field Programmable Gate Array (FPGA) chips, and ADC chips. Each digital board provides power for two ladders, configures and controls two analog boards in the ladders, and carries out digitization of analog signals from the two ladders. The digital board for the ISS-CREAM SCD have been modified from ones for the balloon-borne experiment to accommodate radiation-hard components (in general larger than no radiation-hard components) and implement the protection measure for single event latch-up for ADC chips while the functionality is kept unchanged.

\subsection{Mechanical Structure}

The supporting mechanical structure is designed in such a fashion to minimize material in front of silicon sensors and to guarantee the effective heat conduction between major heat dissipating electronics components and mechanical structure. There are twenty four ladders and twelve digital boards installed in each SCD layer. The single layer layout is shown in Fig. 2 where all twelve digital boards are fully installed, but the ladders are not fully installed. The layout shows three completely empty slots with no ladders, three slots with only analog boards placed, and one slot with three silicon sensors removed. A ladder is a basic unit that populates the active area, and the silicon sensors are never removed from the analog board in the ladder once the ladder is mounted. Therefore, in the single layer layout of Fig. 2, the partial or full front sides of four analog boards (in green) are shown only for illustration purpose to aid the reader's understanding. 


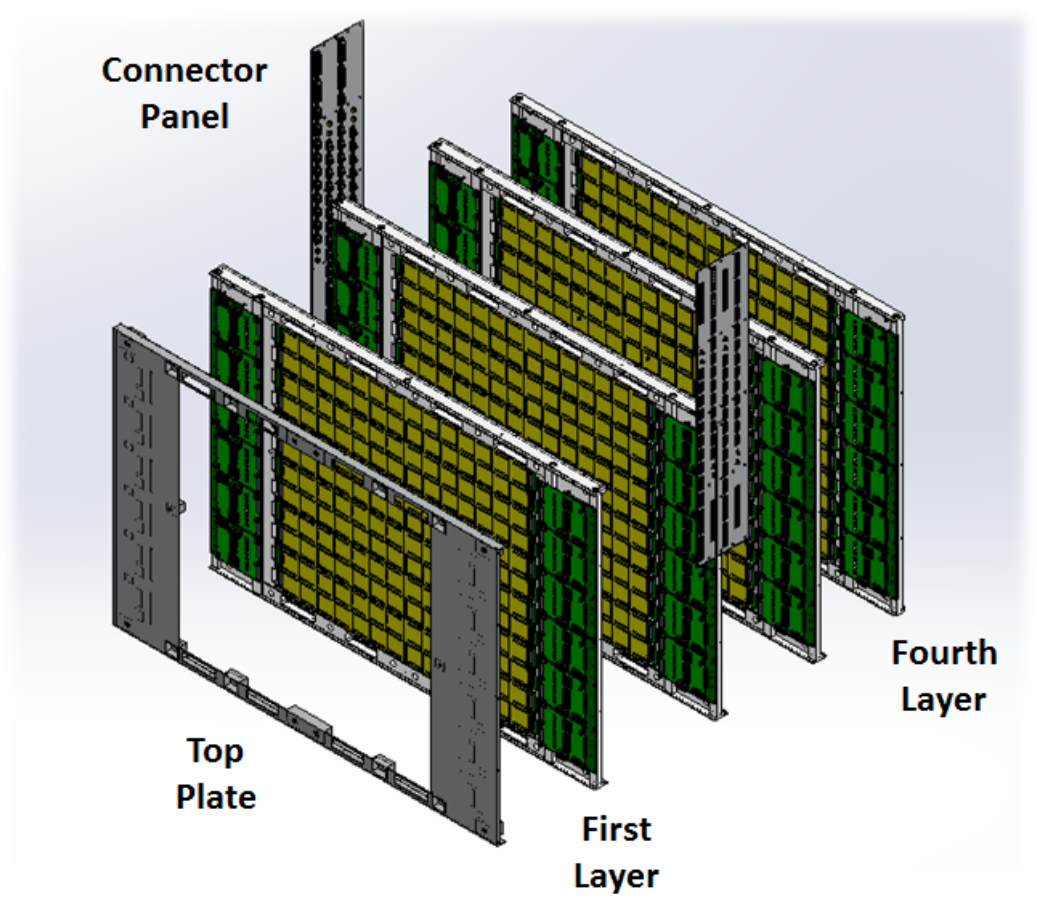

Figure 3: SCD layers along with top plate and connector panels.

Shown in Fig. 3 are the four SCD layers, top plate and two connector panels. The assembly order of the SCD is as follows: The fourth layer and the connector panels are assembled together first. Then the third layer is place over the fourth layer, the second layer over the third layer, and the first layer over the second layer. Finally the top plate is placed over the first to complete the SCD assembly. Note that the top plate is open at the active area to minimize material in front of silicon sensors. Also note that the top plate has ten 3/8-inch fastener holes where the SCD is fixed to the ISS-CREAM payload.

\section{Space Environment Tests of the SCD}

All electrical and mechanical components of the SCD have been selected and manufactured in compliance with the workmanship and procedure required by the Safety and Mission Assurance standard of NASA. Then the SCD has undergone space qualification tests, namely thermal vacuum test and vibration test, for the verification of its suitability and performance assurance for the ISS-CREAM mission. These space environment tests have been carried out according to the requirement and procedure described in the CREAM Subsystem Verification Plan [6] which is the ISS-CREAM-tailored version of General Environmental Verification Standard of NASA, GSFCSTD-7000A [7].

Each layer of the SCD went through the thermal vacuum test to verify that it should operate and function with no failure in the space thermal vacuum environment. All layers of the SCD operated at hot and cold plateaus of thermal cycles with no failure, and there was no difference in the performance of the SCD at the ambient environment and the thermal vacuum environment. 


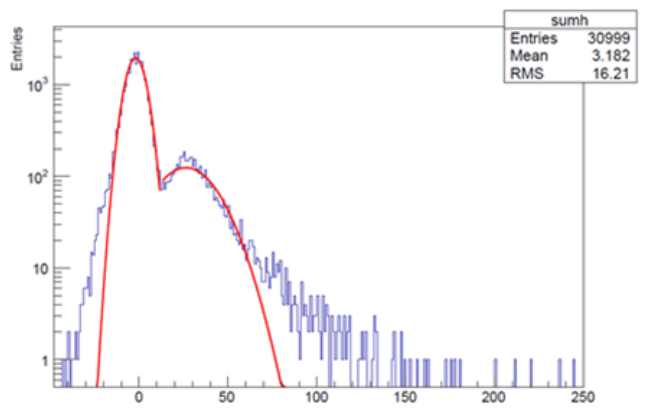

Pedestal Subtracted ADC distribution

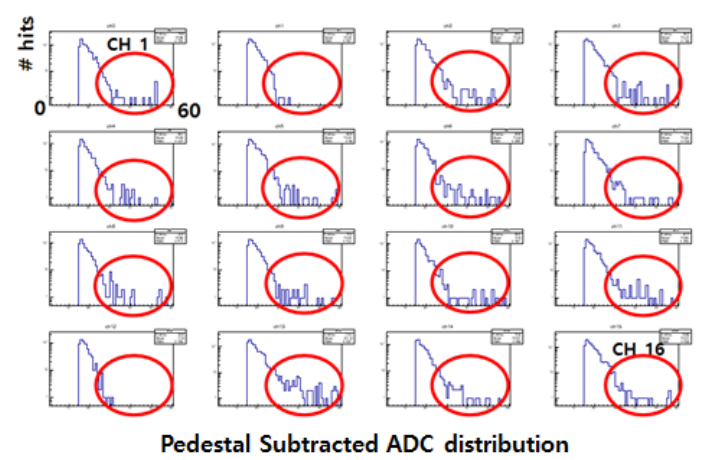

Pedestal Subtracted ADC distribution

Figure 4: ${ }^{90} \mathrm{Sr}$ electron signals observed in a SCD channel (left) and muon signals, marked by red circles, observed in the 16 channels of one SCD sensor (right).

The four-layer SCD was fully assembled after the thermal vacuum test of all single layers, and the SCD assembly underwent the vibration test. Functional tests of the SCD assembly were carried out before and after the vibration test to compare the SCD performance, and no difference was observed in the SCD performance before and after the vibration test.

\section{Performance of the SCD}

Before the assembly of the SCD, the SCD channels were tested with a ${ }^{90} \mathrm{Sr}$ beta source. The typical response of the SCD channels to the beta source is shown in the left of Fig. 4. From the fit shown in red, the pedestal mean and width are $-1.93 \pm 0.03$ and $5.29 \pm 0.03$, respectively, and the peak value of the beta source signal is $26.33 \pm 0.52$. Therefore the typical signal to noise ratio of the SCD measured with the beta source is $\sim 5$. After the SCD was integrated into the ISS-CREAM payload, the SCD was tested in-between space environment tests with the muon events triggered by the external scintillators placed top and bottom of the payload. An example of the muon events observed in the SCD is shown in the right of Fig. 4.

\section{Conclusion}

All single layers of the SCD completed the thermal vacuum test with no failure found, and the fully assembled four-layer SCD passed the instrument-level vibration test successfully. The SCD was integrated into the ISS-CREAM payload and the payload is undergoing the system-level space environment tests. The performance of the SCD during integration has been more than satisfactory to ensure we can meet our mission requirements.

\section{Acknowledgements}

The authors thank NASA GSFC WFF for project management and engineering support, and NASA JSC ISS Program Office for launch support and the ISS accommodation. This work was supported in the U.S. by NASA grants NNX11AC52G, NNX08AC15G, NNX08AC16G and their predecessor grants, as well as by directed RTOP funds to NASA GSFC WFF. It is supported in Korea 
by the Creative Research Initiatives of MEST/NRF, by the Defense Acquisition Program Administration (DAPA) and by the Agency for Defense Development (ADD) in Korea (ADDR-117), and by National Research Foundation Grants NRF-2014R1A2A2A01002734, NRF-2014R1A1A2006456. It is supported in France by IN2P3/CNRS and CNES and in Mexico by DGAPA-UNAM and CONACYT. The authors also thank H. S. Choi, Korea Institute of Industrial Technology, for contributions to the SCD thermal vacuum tests, M. Geske, Penn State, for contributions to the BSD, and M. A. Coplan, University of Maryland, contributions to CAL electronics vacuum tests.

\section{References}

[1] H. S. Ahn et al., Discrepant hardening observed in cosmic-ray elemental spectra, Astrophys. J 714 (2010), L89-L93.

[2] E.S. Seo et al., Cosmic Ray Energetics And Mass for the International Space Station (ISS-CREAM), Advances in Space Research 53 (2014), 1451-1455 and references therein.

[3] I .H. Park et al., Silicon charge detector for the CREAM experiment, Nucl. Instrum. Methods A 570 (2007), 286-291.

[4] J. H. Adams et al., The CR-1 chip: custom VLSI circuitry for cosmic rays, Proceedings of the 26th International Cosmic Ray Conference, Salt Lake City, Vol. 5 (1999), 69-71.

[5] M. Boezio et al., A high granularity imaging calorimeter for cosimic-ray physics, Nucl. Instrum. Methods A 487 (2002), 407-422.

[6] L. Thompson et al., CREAM Subsystem Verification Plan, ISS-CREAM CVP-101, ISS-CREAM Collaboration internal document (2013), 1-51.

[7] NASA Goddard Space Flight Center, General Environmental Verification Standard For GSFC Flight Programs and Projects, GSFC-STD-7000A, https://standards.nasa.gov/documents/gsfc (2013), 1-203. 